\title{
Multidimensional analysis of the host response reveals prognostic and pathogen-driven immune subtypes among adults with sepsis in Uganda
}

Matthew J. Cummings ${ }^{1,2^{*}}\left(\mathbb{0}\right.$, Barnabas Bakamutumaho ${ }^{3,4}$, Adam Price $^{2}$, Nicholas Owor ${ }^{3}$, John Kayiwa ${ }^{3}$, Joyce Namulondo ${ }^{3}$, Timothy Byaruhanga ${ }^{3}$, Moses Muwanga ${ }^{5}$, Christopher Nsereko ${ }^{5}$, Stephen Sameroff ${ }^{2}$, Rafal Tokarz ${ }^{2}$, Wai Wong ${ }^{2}$, Shivang S. Shah ${ }^{6}$, Michelle H. Larsen ${ }^{7}$ W. Ian Lipkin ${ }^{2,8,9}$, Julius J. Lutwama ${ }^{3}$ and Max R. O'Donnell ${ }^{1,2,9}$

\begin{abstract}
Background: The global burden of sepsis is concentrated in sub-Saharan Africa, where severe infections disproportionately affect young, HIV-infected adults and high-burden pathogens are unique. In this context, poor understanding of sepsis immunopathology represents a crucial barrier to development of locally-effective treatment strategies. We sought to determine inter-individual immunologic heterogeneity among adults hospitalized with sepsis in a subSaharan African setting, and characterize associations between immune subtypes, infecting pathogens, and clinical outcomes.

Methods: Among a prospective observational cohort of 288 adults hospitalized with suspected sepsis in Uganda, we applied machine learning methods to 14 soluble host immune mediators, reflective of key domains of sepsis immunopathology (innate and adaptive immune activation, endothelial dysfunction, fibrinolysis), to identify immune subtypes in randomly-split discovery $(N=201)$ and internal validation $(N=87)$ sub-cohorts. In parallel, we applied similar methods to whole-blood RNA-sequencing data from a consecutive subset of patients $(N=128)$ to identify transcriptional subtypes, which we characterized using biological pathway and immune cell-type deconvolution analyses.

Results: Unsupervised clustering consistently identified two immune subtypes defined by differential activation of pro-inflammatory innate and adaptive immune pathways, with transcriptional evidence of concomitant CD56(-)/ CD16(+) NK-cell expansion, T-cell exhaustion, and oxidative-stress and hypoxia-induced metabolic and cell-cycle reprogramming in the hyperinflammatory subtype. Immune subtypes defined by greater pro-inflammatory immune activation, T-cell exhaustion, and metabolic reprogramming were consistently associated with a high-prevalence of severe and often disseminated HIV-associated tuberculosis, as well as more extensive organ dysfunction, worse functional outcomes, and higher 30-day mortality.
\end{abstract}

\footnotetext{
*Correspondence: mjc2244@columbia.edu

${ }^{1}$ Division of Pulmonary, Allergy, and Critical Care Medicine, Department

of Medicine, Columbia University Vagelos College of Physicians

and Surgeons, 622 West 168th St, PH 8E-101, New York, NY 10032, USA

Full list of author information is available at the end of the article
}

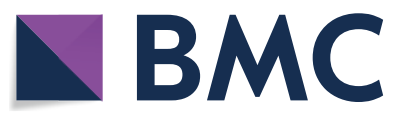

(c) The Author(s) 2022. Open Access This article is licensed under a Creative Commons Attribution 4.0 International License, which permits use, sharing, adaptation, distribution and reproduction in any medium or format, as long as you give appropriate credit to the original author(s) and the source, provide a link to the Creative Commons licence, and indicate if changes were made. The images or other third party material in this article are included in the article's Creative Commons licence, unless indicated otherwise in a credit line to the material. If material is not included in the article's Creative Commons licence and your intended use is not permitted by statutory regulation or exceeds the permitted use, you will need to obtain permission directly from the copyright holder. To view a copy of this licence, visit http://creativecommons.org/licenses/by/4.0/. The Creative Commons Public Domain Dedication waiver (http://creativeco mmons.org/publicdomain/zero/1.0/) applies to the data made available in this article, unless otherwise stated in a credit line to the data. 
Conclusions: Our results highlight unique host- and pathogen-driven features of sepsis immunopathology in subSaharan Africa, including the importance of severe HIV-associated tuberculosis, and reinforce the need to develop more biologically-informed treatment strategies in the region, particularly those incorporating immunomodulation.

Keywords: Sepsis, Biomarkers, Tuberculosis, High-throughput nucleotide sequencing, Uganda, Africa

\section{Introduction}

The global burden of sepsis is concentrated in sub-Saharan Africa (SSA), where nearly $40 \%$ of all sepsis cases occur and up to $65 \%$ of all deaths are sepsis-related [1, 2]. In this context, where epidemic HIV, extensive pathogen diversity, and limited critical care capacity challenge effective management of life-threatening infections, clinical trials of sepsis treatment protocols developed in highincome countries (HICs) have repeatedly shown harm $[3,4]$. As treatment efficacy likely depends, in part, on modifying complex host responses incited by an array of pathogens, imprecise understanding of biological heterogeneity inherent to sepsis in SSA represents a crucial barrier to development of locally-effective management strategies [5].

In high-income countries (HICs), where sepsis typically affects older adults with severe bacterial infections [2], comprehensive profiling of the host response has established immunopathological models defined by dynamic features of inflammation, dysregulated endothelial and cellular metabolic function, and immunosuppression. Although identification of effective, biologically-driven therapeutics for sepsis in HICs remains elusive, such models have laid the conceptual groundwork for development of more precise treatment strategies [5-10]. In SSA, where sepsis disproportionately affects young, HIVinfected adults and high-burden pathogens are unique [3], data informing locally-relevant models of sepsis immunopathology are scarce.

Among a prospective cohort of adults hospitalized with suspected sepsis in Uganda [11], we applied a multidimensional approach to determine inter-individual immunologic heterogeneity and identify distinct, biologically-driven host response subtypes that may have prognostic and therapeutic relevance in the unique SSA context.

\section{Methods}

\section{Study setting, participants, and design}

In this exploratory study, we analyzed data and blood samples from a prospective observational cohort (Research in the Epidemiology of Severe and Emerging Infections in Uganda; RESERVE-U) of adults (age $\geq 18$ years) hospitalized with severe, undifferentiated infection (suspected sepsis) at Entebbe General Referral Hospital (EGRH) in central Uganda from April
2017-August 2019 [11]. EGRH is a 200-bed public district referral hospital with a catchment area of approximately 3 million persons. In the primary catchment area, HIV prevalence is approximately $6 \%$ and malaria is endemic [11]. Representative of a general district hospital in SSA, there is no intensive care unit at EGRH. No vasopressor or inotropic agents are available and intravenous (IV) fluid is typically delivered as $250-500 \mathrm{ml}$ infusions of crystalloid. As no piped oxygen was available at EGRH during the enrollment period, oxygen concentrators were provided to hospital wards as part of the study program.

Patients were included in the parent RESERVE-U study if they fulfilled the following criteria: (1) age $\geq 18$ years, (2) reported a history of fever or had a recorded axillary temperature of $\geq 37.5^{\circ} \mathrm{C}$ at presentation, (3) had clinical illness severe enough to warrant admission to hospital, and (4) were able to provide informed consent or had a surrogate available to do so. Patients were excluded if they presented following trauma or were admitted to a non-medical ward. Study enrollment occurred within $24 \mathrm{~h}$ of hospital admission. Further details of the RESERVE-U study have been published and are summarized in the supplement [11].

Among patients enrolled in the parent RESERVE-U study, we applied a multidimensional approach to dissect the host immune response (Additional file 1: Figure E1). First, given the unsupervised nature of our analyses, we randomly split our parent cohort of 288 patients into discovery $(70 \% ; \mathrm{N}=201)$ and internal validation $(30 \%$; $\mathrm{N}=87$ ) sub-cohorts. We then performed unsupervised clustering on 14 soluble host mediators, chosen a priori to reflect putative domains of sepsis immunopathology (innate and adaptive immune activation, endothelial dysfunction, fibrinolysis), to identify immune subtypes in the discovery and internal validation cohorts independently. Molecular signatures defining each immune subtype in the discovery cohort were explored using classification, regression, and network analyses. In parallel, we performed unsupervised clustering on whole-blood RNA-sequencing data from a consecutive subset of 128 patients in the parent cohort to identify transcriptional subtypes, which we characterized using biological pathway and immune cell-type deconvolution analyses. We compared microbiological characteristics and clinical outcomes across soluble mediator- and transcriptionallyderived immune subtypes, and integrated these results 
to harmonize the biological and clinical relevance of our findings.

\section{Pathogen diagnostics}

For all enrolled patients in the RESERVE-U study, rapid testing was performed for malaria, influenza, and HIV; for HIV-infected patients testing for tuberculosis (TB) was also performed. Testing for these pathogens was informed by World Health Organization (WHO) guidelines for management of sepsis and septic shock in resource-limited hospitals in sub-Saharan Africa (WHO Integrated Management of Adolescent and Adult Illness [IMAI] District Clinician Manual) [12]. The WHO IMAI guidelines emphasize rapid testing for malaria and HIV, a low threshold for TB testing among HIV-infected patients, and consideration of testing or empiric treatment for influenza. Further details are in the supplement.

\section{Serum immunoassays}

From cryopreserved serum samples collected at the time of study enrollment, interleukin (IL)-6, IL-8, IL-10, interferon (IFN) $-\gamma$, IFN- $\gamma$-induced protein-10/C-X-C motif chemokine 10 (IP-10/CXCL10), macrophage inflammatory protein-1-alpha/chemokine $(\mathrm{C}-\mathrm{C}$ motif) ligand 3 (MIP-1 $\alpha / C C L 3)$, macrophage inflammatory protein1-beta/chemokine (C-C motif) ligand 4 (MIP-1 $\beta / C C L 4)$, tumor necrosis factor-alpha (TNF- $\alpha$ ), angiopoietin-1 (Ang-1), angiopoietin-2 (Ang-2), macrophage migration inhibitory factor (MIF), plasminogen activator inhibitor-1 (PAI-1), soluble TNF-receptor type 1 (sTNFR1), and soluble IL-2 receptor alpha/soluble CD25 (sIL2RA/sCD25), were quantified using custom Luminex 200 system kits (Luminex, Austin, TX, USA) from MilliporeSigma (Burlington, Massachusetts, USA) and R\&D Systems (Minneapolis, MN, USA). Further details are in the supplement.

\section{Whole-blood RNA isolation, library preparation, and sequencing}

From cryopreserved whole-blood samples collected in PAXgene blood RNA tubes (PreAnalytiX, Qiagen/ BD, Hombrechtikon, Switzerland) at the time of study enrollment, RNA was isolated and purified using PAXgene blood RNA kits (Qiagen, Hilden, Germany). RNA sequencing libraries were prepared using the NEBNext Ultra RNA Library Prep Kit (NEB, Ipswich, MA, USA). Sequencing libraries were multiplexed and analyzed using a $2 \times 150$ paired-end configuration on the Illumina HiSeq 4000 platform (Illumina, Inc., San Diego, CA, USA). Adapters were trimmed from raw reads using Trimmomatic and sequencing data quality was assessed with FastQC $[13,14]$. Sequencing reads were aligned to the human genome (GRCh38) using STAR and transcript quantification was performed using the R-subread package's featureCounts utility $[15,16]$. Further details are in the supplement.

\section{Analysis of soluble immune mediators}

To identify immune subtypes, we applied unsupervised clustering methods to serum mediator concentrations from patients in the discovery and internal validation cohorts independently. Clustering is a form of unsupervised machine learning that seeks to classify individual data points into clusters, or groups, based on metrics of similarity (e.g., distance, correlation), when the underlying group structure is unknown [17]. Specifically, we applied agglomerative hierarchical clustering (using Ward's method and Euclidean distance) to $\log _{10}$ transformed, scaled, and centered mediator concentrations to initiate a cluster partition, followed by a k-means procedure to consolidate cluster membership [18]. We used within-cluster-sum-of-squares to determine the optimal number of clusters, which we confirmed using over 20 indices of cluster validity and stability [19]. We visualized between-cluster variance in mediator concentrations using principal component analysis and standardized heatmaps. Further details are in the supplement.

To identify the most influential variables driving cluster (i.e. immune subtype) assignment in the discovery cohort, we determined representation of mediator variables on the first two principal components by calculating squared factor loadings for each variable. Separately, we applied a gradient-boosted decision tree algorithm, trained to predict cluster assignment, to our mediator variables, and identified the most important discriminatory variables using their respective split gain values. Next, to explore longitudinal, cluster-specific changes in soluble mediators over the course of illness, we evaluated the relationship between mediator concentrations and reported duration of illness at admission (obtained via patient or surrogate) using robust regression, with patient-level datapoints stratified by cluster assignment. Lastly, to determine inter-mediator relationships within each discovery cohort cluster and identify "central" mediators around which each cluster may be coordinated, we constructed force-directed weighted correlation networks [20-22]. Further details are in the supplement.

\section{Analysis of whole-blood RNA-sequencing data}

Independent of soluble mediator analyses and using the same parameters, we performed unsupervised hierarchical clustering of whole-blood RNA-sequencing data from a consecutively enrolled subset of patients $(\mathrm{N}=128)$ in the RESERVE-U cohort. Following determination of the optimal number of clusters (i.e. transcriptional subtypes) using within-cluster-sum-of-squares, 
we identified genes that were differentially expressed across each cluster based on a log-fold change $\geq|1|$ and Benjamini-Hochberg-adjusted $\mathrm{p}$-value $\leq 0.01$ [23, 24]. Differentially expressed gene sets were selected for biological pathway analysis (Ingenuity Pathway Analysis, Qiagen), results of which were examined to infer functional differences between identified clusters. Using the ImmQuant software package and IRIS and DMAP mRNA compendiums, we applied digital cell quantification (DCQ) deconvolution to our differentially expressed gene sets to infer relative immune cell quantities across transcriptional clusters [25-27]. Further details are in the supplement.

\section{Statistical analyses}

In presentations of clinical data, continuous variables are expressed as medians (interquartile range [IQR]) and categorical variables are summarized as counts and percentages with $95 \%$ confidence intervals (CI) and twosided p-values presented where relevant. Clinical and microbiological characteristics across immune mediator- and transcriptional subtypes were compared using Chi-squared, Fisher exact, or Wilcoxon rank-sum tests as appropriate. Univariable and multivariable logistic regression were used to compare the primary outcome of 30-day mortality across identified immune subtypes. Given the exploratory nature of this study, no adjustment for multiple comparisons was performed unless indicated. For the primary outcome of 30-day vital status, mortality was not imputed if vital status was unknown, and a sensitivity analysis was performed to account for potential bias due to loss-to-follow-up. Further details are in the supplement.

Analyses were performed using R (v3.6.1, R Foundation for Statistical Computing, Vienna, Austria) via the RStudio (v1.4.1106) environment, with specific packages detailed in the supplement. Biological pathway and immune deconvolution analyses of RNA-sequencing data were performed using Ingenuity Pathway Analysis (Qiagen, Hilden, Germany) and ImmQuant, respectively [25]. Study overview and flow diagrams were created with Biorender.

\section{Results}

\section{Participants}

Of 301 adult patients enrolled in the RESERVE-U cohort, 288 (96\%) and 128 (43\%) had serum and wholeblood RNA samples available for analysis, respectively, and were included in this study (Additional file 1: Figure E2). Characteristics of the 288 patients for whom soluble mediators were analyzed, nearly $90 \%$ of whom had $\geq 1$ quick-Sepsis-related Organ Failure Assessment
(qSOFA) criterion, are presented in Table 1. Characteristics of patients who had RNA samples analyzed were similar to those who did not (Additional file 1: Table E1).

\section{Unsupervised clustering of soluble mediators consistently reveals two immune subtypes among adults with suspected sepsis in Uganda}

In the discovery and internal validation cohorts, a two cluster (i.e. two subtype) model was determined to be optimal based on multiple indices of cluster stability and validity (Fig. 1a, b and Additional file 1: Figure E3AE3B, Tables E2-E3), with clear between-cluster separation across the first principal component of mediator variance (Figs. 1c and Additional file 1: Figure E3C). In both the discovery and internal validation cohorts, soluble mediator subtype 2 (S2) was associated with significantly higher concentrations of key mediators promoting inflammation (MIF, IFN- $\gamma$, IL-6, TNF- $\alpha$ ), T-cell activation and tolerance (sIL-2Ra/sCD25), and neutrophil (IL-8), monocyte/macrophage, NK, Th1, and dendritic-cell (IP10/CXCL10, MIP-1 $\alpha /$ CCL3, MIP-1 $\beta / C C L 4)$ chemotaxis (Figs. 1d, Additional file 1: Figure E3D, E4-E5, Tables E4-E5). Although these markers were observed alongside elevated concentrations of anti-inflammatory mediators (sTNFR1 and IL-10), ratios of IL-6/IL-10, IFN- $\gamma$ / IL-10, and TNF- $\alpha /$ sTNFR1 were consistently higher in subtype 2, suggesting imbalance towards a more proinflammatory state (Additional file 1: Figures E4-E5, Tables E4-E5). While concentrations of Ang-1, which promotes endothelial stability and dampening of inflammation, were higher in subtype 1 (S1), those of Ang-2, a pro-inflammatory mediator of endothelial activation and destabilization, were significantly higher in S2, as was the ratio of Ang-2/Ang-1. Examination of squared factor loadings and gradient-boosted decision tree models both identified sTNFR1, IP-10/CXCL10, TNF- $\alpha$, IL-6, and sIL$2 \mathrm{Ra} / \mathrm{sCD} 25$ as the most influential mediators driving subtype assignment (Fig. 1e, f).

\section{Network analyses demonstrate subtype-specific responses coordinated around shared and distinct mediators}

Force-directed network structures were unique to each discovery cohort subtype and were differentially coordinated around a core of central mediators. Although some mediators were central in both subtypes, the core S1 network included more anti-inflammatory and endothelialprotective mediators (sTNFR1, IL-10, Ang-1) (Fig. 1g). In contrast, S2 was coordinated around more pro-inflammatory and chemotactic (MIP-1 $\alpha / C C L 3$, MIP-1 $\beta / C C L 4$, 
Table 1 Patient characteristics stratified by discovery and internal validation cohorts

\begin{tabular}{|c|c|c|c|}
\hline Patient characteristic & All patients $(\mathrm{N}=288)$ & $\begin{array}{l}\text { Discovery cohort } \\
(\mathrm{N}=201)\end{array}$ & $\begin{array}{l}\text { Internal } \\
\text { validation cohort } \\
(\mathrm{N}=87)\end{array}$ \\
\hline Female sex, n (\%) & $171 / 288(59.4)$ & $116 / 201(57.7)$ & $55 / 87(63.2)$ \\
\hline Age, years, median $[\mathrm{IQR}]$ & $32[26,42]$ & $32[26,40]$ & $32[27,43]$ \\
\hline Duration of illness prior to admission, days, median $[\mathrm{IQR}]^{\mathrm{a}}$ & $4[3,7]$ & $4[3,7]$ & $4[3,7]$ \\
\hline History of fever, n (\%) & $288 / 288(100.0)$ & $201 / 201(100.0)$ & $87 / 87(100.0)$ \\
\hline Night sweats & $225 / 288(78.1)$ & $161 / 201(80.1)$ & $64 / 87(73.6)$ \\
\hline Headache & $227 / 288(78.8)$ & $158 / 201(78.6)$ & $69 / 87(79.3)$ \\
\hline Cough & $178 / 288(61.8)$ & $125 / 201(62.2)$ & $53 / 87(60.9)$ \\
\hline Diarrhea & $100 / 288(34.7)$ & $70 / 201(34.8)$ & $30 / 87(34.5)$ \\
\hline Shortness of breath & $66 / 288(22.9)$ & 47/201 (23.4) & 19/87 (21.8) \\
\hline Dysuria & $39 / 288(13.5)$ & $31 / 201(15.4)$ & 8/87 (9.2) \\
\hline Received antibiotic or antimalarial agent prior to admission, $\mathrm{n}(\%)$ & $102 / 288(35.4)$ & $70 / 201(34.8)$ & $32 / 87(36.8)$ \\
\hline Temperature $\geq 38^{\circ} \mathrm{C}, \mathrm{n}(\%)$ & $104 / 288(36.1)$ & 76/201 (37.8) & $28 / 87(32.2)$ \\
\hline Temperature $<36{ }^{\circ} \mathrm{C}, \mathrm{n}(\%)$ & $84 / 288(29.2)$ & $58 / 201(28.9)$ & 26/87 (29.9) \\
\hline Heart rate, beats/min, median [IQR] & $98[87,109]$ & $98[86,108]$ & $98[90,111]$ \\
\hline Respiratory rate, beats/min, median [IQR] & $22[21,26]$ & $22[21,26]$ & $22[20,26]$ \\
\hline Systolic blood pressure, mmHg, median [IQR] & $103[91,117]$ & $104[91,118]$ & $100[92,113]$ \\
\hline Oxygen saturation, \%, median [IQR] & $97[95,98]$ & $97[96,98]$ & $97[95,98]$ \\
\hline Encephalopathy, n (\%) ${ }^{b}$ & $57 / 288(19.8)$ & $40 / 201(19.9)$ & $17 / 87(19.5)$ \\
\hline qSOFA score $\geq 2, n(\%)^{c}$ & $129 / 288(44.8)$ & $88 / 201(43.8)$ & $41 / 87(47.1)$ \\
\hline qSOFA score $\geq 1, \mathrm{n}(\%)^{c}$ & $253 / 288(87.8)$ & $174 / 201(86.6)$ & $79 / 87(90.8)$ \\
\hline Modified SIRS score $\geq 2, n(\%)^{d}$ & $247 / 288(85.8)$ & $173 / 201(86.1)$ & $74 / 87(85.1)$ \\
\hline MEWS, median $[\mathrm{IQR}]$ & $3[2,5]$ & $3[2,4]$ & $3[2,5]$ \\
\hline UVA score, median [IQR] & $3[2,4]$ & $3[1,4]$ & $2[2,4]$ \\
\hline Shock, n (\%) & $41 / 288(14.2)$ & 28/201 (13.9) & $13 / 87(14.9)$ \\
\hline Acute respiratory failure, $\mathrm{n}(\%)^{\mathrm{f}}$ & $61 / 288(21.2)$ & $39 / 201(19.4)$ & $22 / 87(25.3)$ \\
\hline Severe anemia, $n(\%)^{9}$ & $56 / 288(19.4)$ & $39 / 201(19.4)$ & $17 / 87(19.5)$ \\
\hline HIV-infected, n (\%) & $154 / 286(53.8)$ & 106/199 (53.2) & $48 / 87(55.2)$ \\
\hline WHO clinical stage 3 or 4, n (\%) & $125 / 154(81.2)$ & $91 / 106(85.8)$ & $34 / 48(71.0)$ \\
\hline Newly diagnosed HIV-infection, n (\%) & 20/154 (13.0) & 12/106 (11.3) & $8 / 48(16.7)$ \\
\hline On ART prior to admission, $\mathrm{n}(\%)^{\mathrm{h}}$ & $91 / 134(67.9)$ & $63 / 94(67.0)$ & $28 / 40(70.0)$ \\
\hline On TMP-SMX prior to admission, $\mathrm{n}(\%)^{\mathrm{h}}$ & $94 / 134(70.1)$ & $65 / 94(69.1)$ & $29 / 40(72.5)$ \\
\hline Malaria RDT positive, n (\%) & $59 / 283(20.8)$ & $38 / 197(19.3)$ & $21 / 86(24.4)$ \\
\hline Microbiological TB positive, n (\%) ${ }^{i}$ & $51 / 288(17.7)$ & $35 / 201(17.4)$ & $16 / 87(18.4)$ \\
\hline Urine TB-LAM positive & $40 / 122(32.8)$ & $27 / 83(32.5)$ & $13 / 39(33.3)$ \\
\hline Influenza PCR positive, n (\%) & $17 / 262(6.5)$ & $14 / 184(7.6)$ & $3 / 78(3.8)$ \\
\hline Death in-hospital or transfer, n (\%) & $40 / 288(13.9)$ & 28/201 (13.9) & $12 / 87(13.8)$ \\
\hline Duration of hospitalization, days, median [IQR] $]^{j}$ & $5[3,8]$ & $5[3,7]$ & $5[3,8]$ \\
\hline KPS $\leq 70$ at alive discharge, $\mathrm{n}(\%)$ & $20 / 246(8.1)$ & $12 / 173(6.9)$ & $8 / 73(11.0)$ \\
\hline Death at 30-days post-discharge, n (\%) & $62 / 260(23.8)$ & $44 / 179(24.6)$ & $18 / 81(22.2)$ \\
\hline
\end{tabular}

IQR: interquartile range, qSOFA: quick sequential (sepsis-related) organ failure assessment, SIRS: systemic inflammatory response syndrome, MEWS: modified early warning score, UVA: universal vital assessment, HIV: human immunodeficiency virus, WHO: World Health Organization, ART: anti-retroviral therapy, RDT: rapid diagnostic test, TB: tuberculosis, LAM: lipoarabinomannan, PCR: polymerase chain reaction

a Unknown for 1 patient

${ }^{b}$ Anything other than "Alert" on AVPU (alert, responsive to voice, responsive to pain, unresponsive) mental status assessment

' Systolic blood pressure $\leq 100 \mathrm{mmHg}$, respiratory rate $\geq 22$ breaths/min, and encephalopathy, latter defined using AVPU scale

d Temperature $\geq 38{ }^{\circ} \mathrm{C}$ or $<36^{\circ} \mathrm{C}$, heart rate $\geq 90$ beats $/ \mathrm{min}$, respiratory rate $\geq 20$ breaths $/ \mathrm{min}$

e Systolic blood pressure $\leq 90 \mathrm{mmHg}$ despite administration of $\geq 1 \mathrm{~L}$ of intravenous fluid

${ }^{f}$ Oxygen saturation $\leq 90 \%$ or respiratory rate $\geq 30$ breaths $/ \mathrm{min}$

${ }^{\mathrm{g}}$ Hemoglobin $<9 \mathrm{~g} / \mathrm{dl}$ or administration of blood transfusion 
Table 1 (continued)

${ }^{\mathrm{h}}$ Denominator is number with known HIV-infection prior to admission

' Positive result by sputum Xpert Ultra or smear or urine TB-LAM

jUnknown for 11 patients

TNF- $\alpha$ ) and endothelial-destabilizing (Ang-2) mediators (Fig. 1h).

\section{Profiles of divergent immune activation and endothelial dysfunction distinguish host subtypes throughout the course of illness}

Nearly all mediators were generally higher in S2 throughout the reported course of illness, with the exception of Ang-1, which appeared consistently higher in S1 (Fig. 2, Additional file 1: Figure E6). While concentrations of many mediators remained stably divergent, there were disproportionate decreases over time for IL-10 and sTNFR1 in S2. In contrast, concentrations of IP-10/ CXCL10, sIL2-Ra/sCD25, and Ang-2 disproportionately increased over time in $\mathrm{S} 2$.

\section{Immune subtypes are associated with differential} profiles of physiologic derangement, organ dysfunction, and mortality

In both the discovery and internal validation cohorts, Universal Vital Assessment (UVA) and Modified Early Warning Scores (MEWS), physiologic indices of clinical severity feasible for use in resource-limited settings, were significantly higher among patients in S2, as were proportions of patients with $\geq 2$ qSOFA criteria (Fig. 3ac, Additional file 1: Tables E2-E3). Consistently, patients in S2 also had more extensive organ dysfunction, including higher prevalence of shock, acute respiratory failure, severe anemia, and encephalopathy (Fig. 3d, Additional file 1: Tables E2-E3).

Given consistent biological and clinical findings in the discovery and internal validation cohorts, we analyzed in-hospital and 30-day outcomes in a pooled sample of patients from both cohorts. In this analysis, mortality at 30-days was significantly higher among patients in S2 vs. S1 (34.6\% vs. $16.3 \%, \mathrm{p}=0.001)$ (Fig. $3 \mathrm{~g}$ ). This finding was consistent when patients with indeterminate 30-day vital status were considered deceased $(39.7 \%$ vs. $25.6 \%$, $\mathrm{p}=0.017$ ) and in multivariable models adjusted for age, sex, duration of illness and multiple indices of clinical severity (Additional file 1: Table E6). In-hospital outcome and discharge functional status among hospital survivors were also significantly worse among patients in S2 (Fig. 3g). When patients in the both the discovery and internal validation cohorts were pooled and stratified by key pathogen groups, estimates of mortality at 30-days were consistently higher among patients in S2 (Fig. 3h).

\section{Immune subtypes are differentiated primarily by severe HIV-associated TB}

Although we observed a range of infections across both subtypes, the prevalence of severe HIV-related infections, including disseminated HIV-associated TB (indicated by positive urine TB-LAM testing) [28], was significantly and consistently higher in S2 compared to S1 (Fig. 3e, f, Additional file 1: Tables E2-E3). Among patients in the discovery and internal validation cohorts with positive urine TB-LAM testing, those in S2 had higher grades of band intensity (median 3 [IQR; 1-3] vs. 1 [IQR 1-3]), which may correspond to higher mycobacterial loads $[29,30]$.

\section{Unsupervised clustering of whole-blood RNA-sequencing data reinforces a two-host subtype partition differentiated by pro-inflammatory innate immune activation, $\mathrm{T}$-cell exhaustion, aberrant NK-cell expansion, and metabolic reprogramming}

In unsupervised hierarchical clustering of whole-blood RNA-sequencing data $(\mathrm{N}=128)$, a two-cluster (i.e.

\footnotetext{
(See figure on next page.)

Fig. 1 Soluble mediator-derived immune subtypes in discovery cohort. a Unsupervised hierarchical clustering of 14 serum mediators reflecting innate and adaptive immune activation, endothelial dysfunction, and fibrinolysis; dendrogram indicates cluster partition prior to k-means consolidation $(\mathrm{N}=201)$. b Optimal cluster partitions suggested by cluster stability and validation indices as per NbClust package. $\mathbf{c}$ First two principal components plotted with the proportion of variance explained by each component; individuals stratified by cluster (subtype) assignment ( $N=201$ ). d Heatmap of z-score standardized soluble mediator concentrations, stratified by cluster (subtype) ( $N=201)$. e Squared factor loadings for all serum mediators across the first two principal components in the discovery cohort; higher loading value indicates greater importance for each variable in explaining variance across each principal component $(\mathrm{N}=201)$. $\mathbf{f}$ Importance of serum mediator variables in construction of gradient-boosted decision tree algorithm designed to predict cluster (subtype) assignment in discovery cohort ( $\mathrm{N}=201$ ); 10 most important variables presented. Force-directed correlation networks based on the Fruchterman-Reingold method in discovery cohort subtype $1 \mathbf{g}$ and subtype $2 \mathbf{h}$; each mediator variable was set as a network node with between-mediator correlations significant at $p$-value $\leq 0.05$ indicated by weighted edges (blue and green edges indicate positive correlation, red edges indicate negative correlation, edge width indicates strength of correlation) $(\mathrm{N}=201)$. Nodes with blue and green shading indicate those mediators considered central in the subtype 1 and 2 networks, respectively, defined as those with $\geq 1$ centrality metric (strength, closeness, or betweenness) above the standardized cluster mean ( $\mathrm{z}$-score $>0$ )
} 
$$
\text { A }
$$

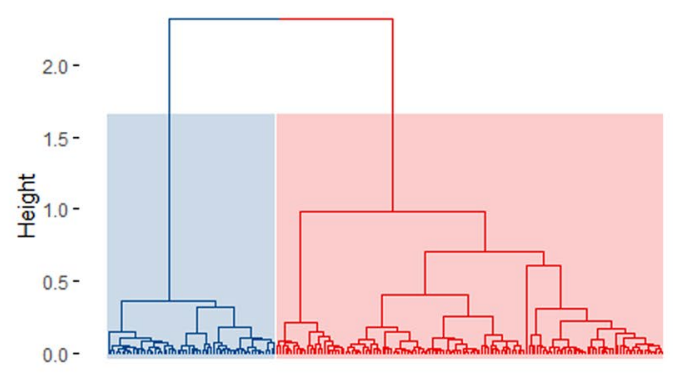

C

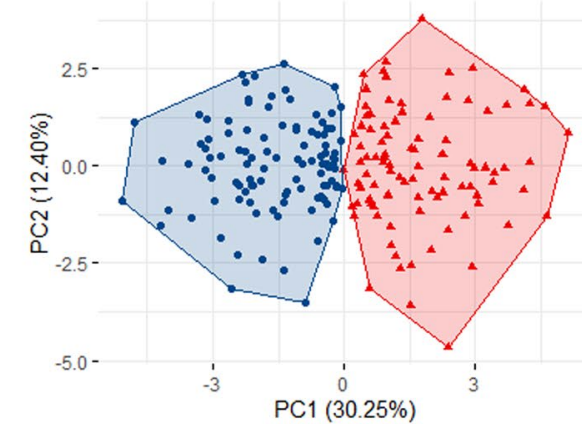

E

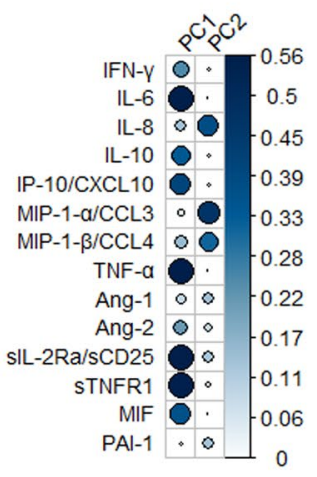

G

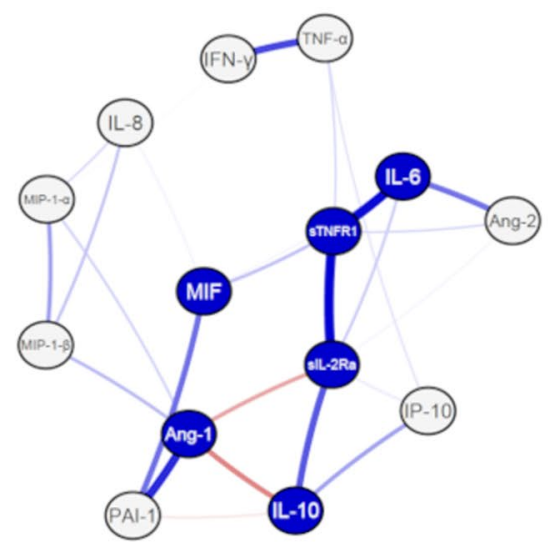

B

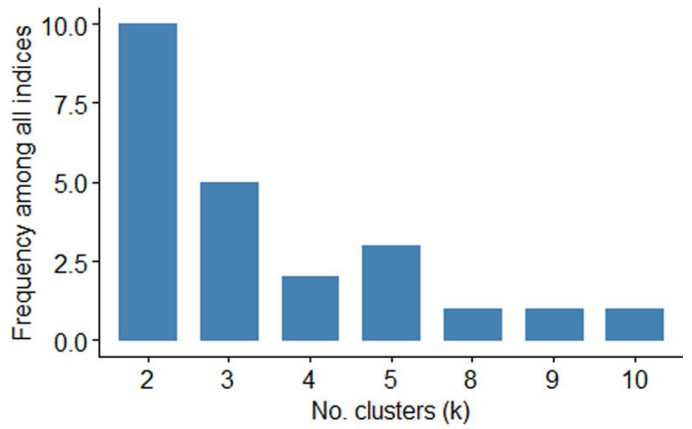

D

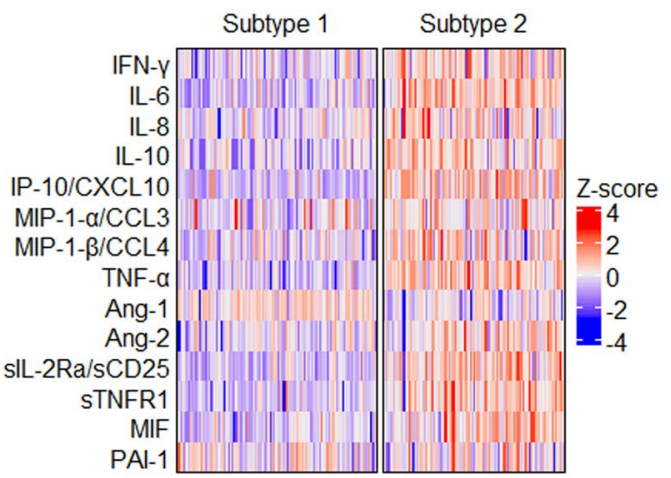

F

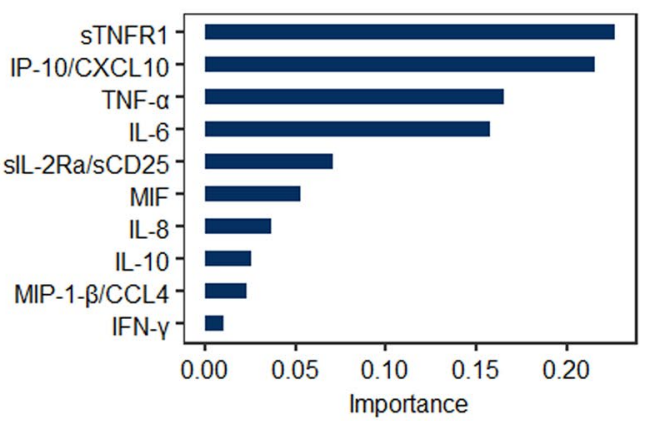

$\mathrm{H}$

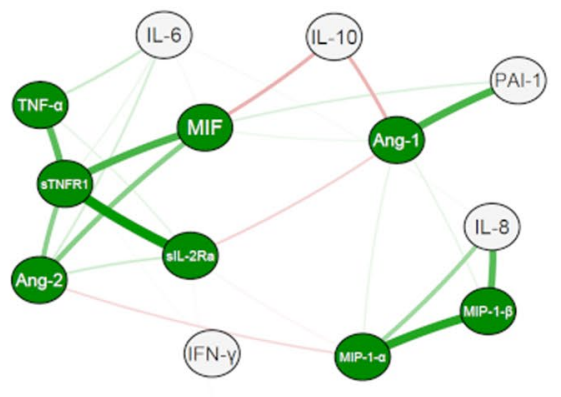

Fig. 1 (See legend on previous page.) 
A

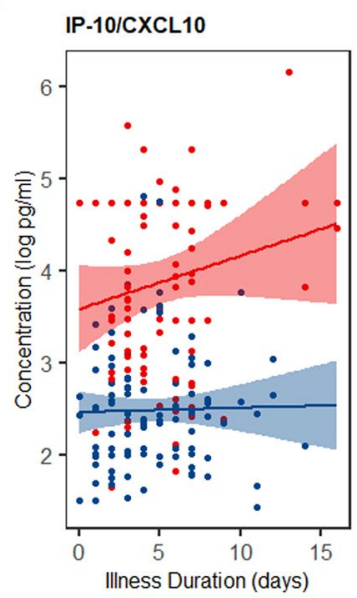

$\mathrm{D}$

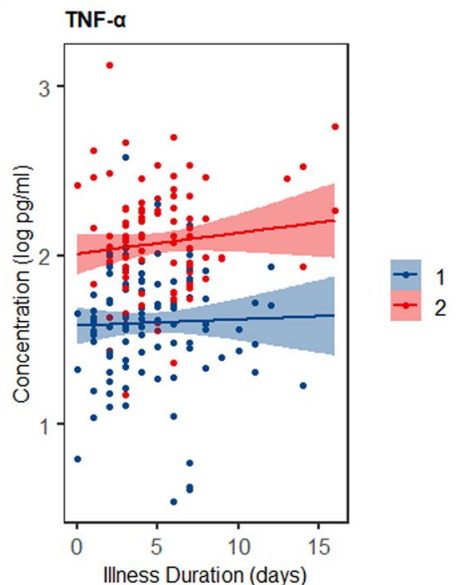

B

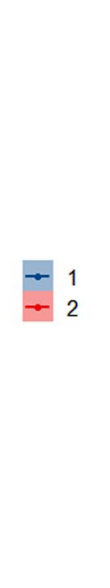

E

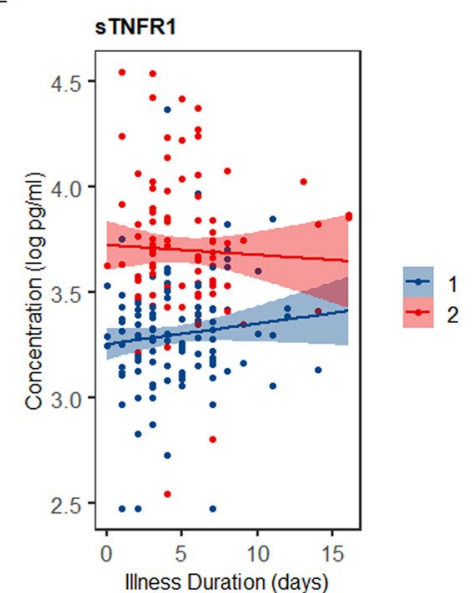

C

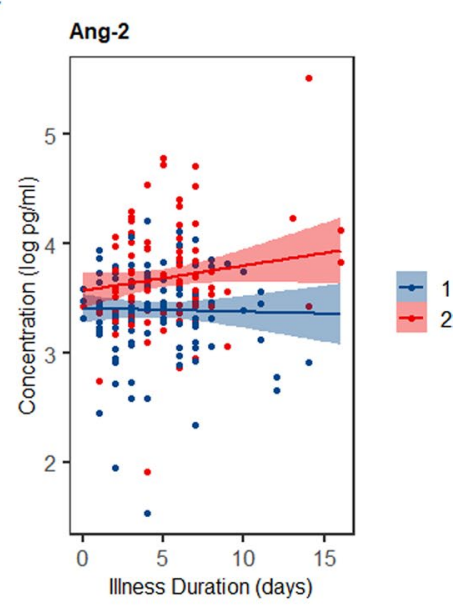

$\mathrm{F}$

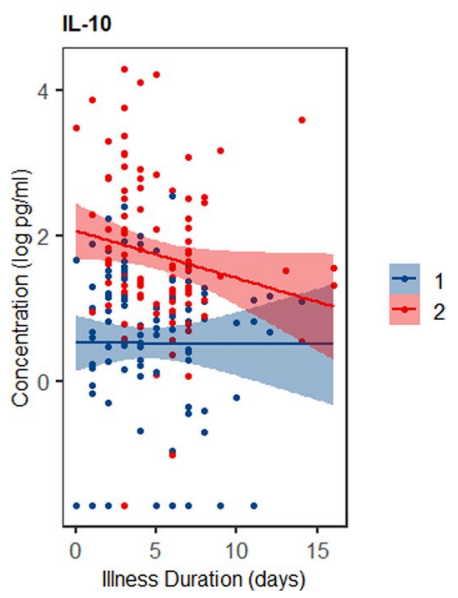

Fig. 2 Serum mediator concentrations over the reported course of illness in the discovery cohort, stratified by immune subtype. a-f

Concentrations of soluble mediators over the reported course of illness, with robust regression lines and $95 \%$ confidence intervals, stratified by immune subtype ( $\mathrm{N}=198 ; 1$ patient with unknown illness duration, 2 patients with extreme outliers in illness duration excluded). For example, an individual data point corresponding to "day 0 " represents the serum mediator concentration for a patient who was admitted to hospital on the day of illness onset, while that corresponding to "day 5" represents a patient who was admitted to hospital on day 5 of illness

transcriptional subtype) model was determined to be optimal (Additional file 1: Figure E7A); 3,561 genes were differentially expressed across subtypes (Additional file 1: Figure E7B). Compared to transcriptional subtype 1 (T1), transcriptional subtype 2 (T2) was characterized by increased expression of genes involved in pathogen recognition, pro-inflammatory cytokine and chemokine signaling, necroptosis, and inflammasome signaling and migration (Fig. 4a). Similar to soluble mediatorderived S2, this marked pro-inflammatory response was accompanied by activation of transcription factor genes (STAT3, PPAR) integral to compensatory, anti-inflammatory immune dampening. Concomitantly, we observed consistent evidence of T-cell exhaustion in T2, including increased expression of genes corresponding to PD-1/ PD-L1 inhibitory checkpoint signaling and decreased expression of genes integral in T-cell receptor signaling (Nur77), activation (CD28, iCos-iCosL, PKC), and proliferation (OX40), and cytotoxic T-cell-mediated apoptosis (Fig. 4b).

Quantitatively, immune cell-type deconvolution analysis inferred increased quantities of pro-inflammatory phagocytes and CD56(-)/CD16(+) NK-cells in T2, with concomitant CD4 and CD8 T-cell depletion (Fig. 4e, f). Notably, CD56(-)/CD16(+) NK-cells are an aberrant, likely exhausted subset that expand during HIV-infection and exhibit limited capacity for cytotoxicity and cytokine (i.e. IFN- $\gamma$ ) production, while retaining capability to 

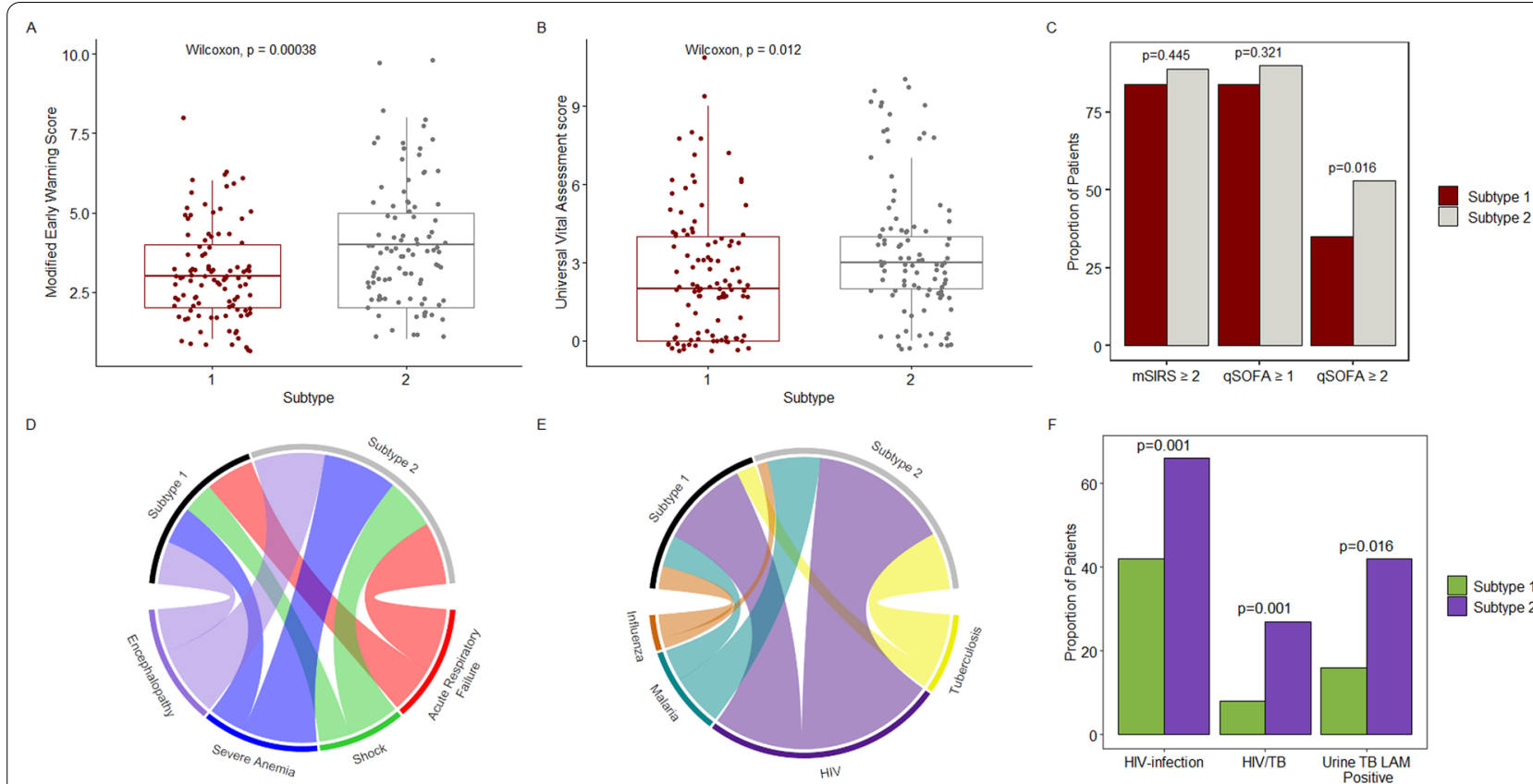

E
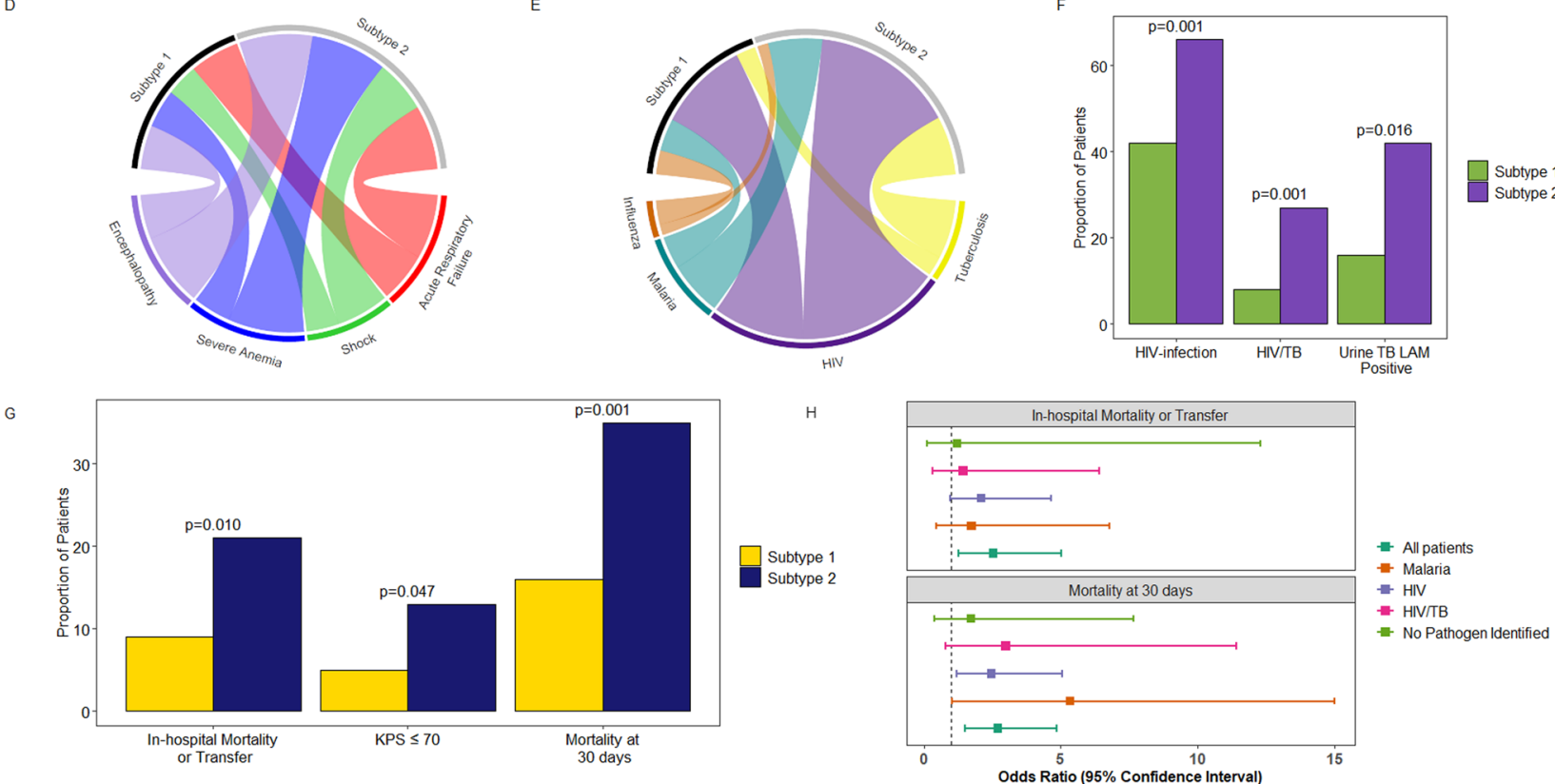

Fig. 3 IIIness severity scores, distributions of organ failures and pathogens, and outcomes stratified by immune subtypes. a-c Modified Early Warning Score, Universal Vital Assessment score, modified systemic inflammatory response syndrome [mSIRS], and quick Sepsis-related Organ Failure assessment [qSOFA] scores stratified by immune subtype in the discovery cohort; p-values in 3C represent Chi-squared test with continuity correction $(\mathrm{N}=201)$. d Chord plot indicating proportion of patients with specific organ failures across each subtype in the discovery cohort; a wider chord band indicates a greater proportion of patients with each corresponding organ failure $(\mathrm{N}=201$, proportions in subtype 2 vs. 1 as follows: shock: $17.7 \%$ vs. $10.5 \%$; acute respiratory failure: $21.9 \%$ vs. $17.1 \%$; severe anemia: $26.0 \%$ vs. $13.3 \%$; encephalopathy: $25.0 \%$ vs. $15.2 \%$ ). e Chord plot indicating proportion of patients with specific infections across each subtype in the discovery cohort; a wider chord band indicates a greater proportion of patients with each corresponding infection ( $N=201$, proportions in subtype 2 vs. 1 as follows: HIV: 65.6\% vs. 42.0\%, tuberculosis: $27.1 \%$ vs. $8.6 \%$, malaria: $24.5 \%$ vs. $14.6 \%$, influenza: $4.5 \%$ vs. $10.5 \%$ ). f Proportions of patients with known HIV-infection status ( $N=199$ ), HIV-associated TB ( $\mathrm{N}=199)$, and positive urine TB-LAM results (among those tested, $\mathrm{N}=83$ ) across each immune subtype in the discovery cohort. g In-hospital outcome ( $N=288)$, impaired functional status [Karnofsky Performance Status; KPS] among hospital survivors $(N=246)$, and 30-day vital status $(N=260)$ across each subtype in a pooled cohort of patients from the discovery and internal validation cohorts; $p$-values in $3 F$ and $3 G$ represent Chi-squared test with continuity correction. $\mathbf{h}$ Forest plot indicating univariable (unadjusted) odds ratios for in-hospital outcome and 30-day mortality among patients in subtype 2 vs. subtype 1, stratified by key pathogen groups in pooled discovery and internal validation cohort [patients with influenza omitted given small number of events in that pathogen group; for visualization, upper limit of $95 \%$ confidence interval for 30-day mortality truncated at 15 for patients with malaria (upper limit 27.57)

secrete pro-inflammatory chemokines such as MIP-1 $\beta$ / CCL4 [31, 32].

Metabolically, patients in T2 showed evidence of arrested cell growth and autophagy (Fig. 4c), with concomitant hypoxia-mediated metabolic reprogramming including a switch to glycolysis and upregulation of bioactive, largely pro-inflammatory lipid mediators (eicosanoids, sphingosine-1-phosphate). Patients in T2 also showed increased expression of genes implicated in oxidative stress (NRF2), endothelial dysfunction and angiogenesis (inducible nitric oxide [iNO], VEGF), with decreased expression of genes corresponding to 

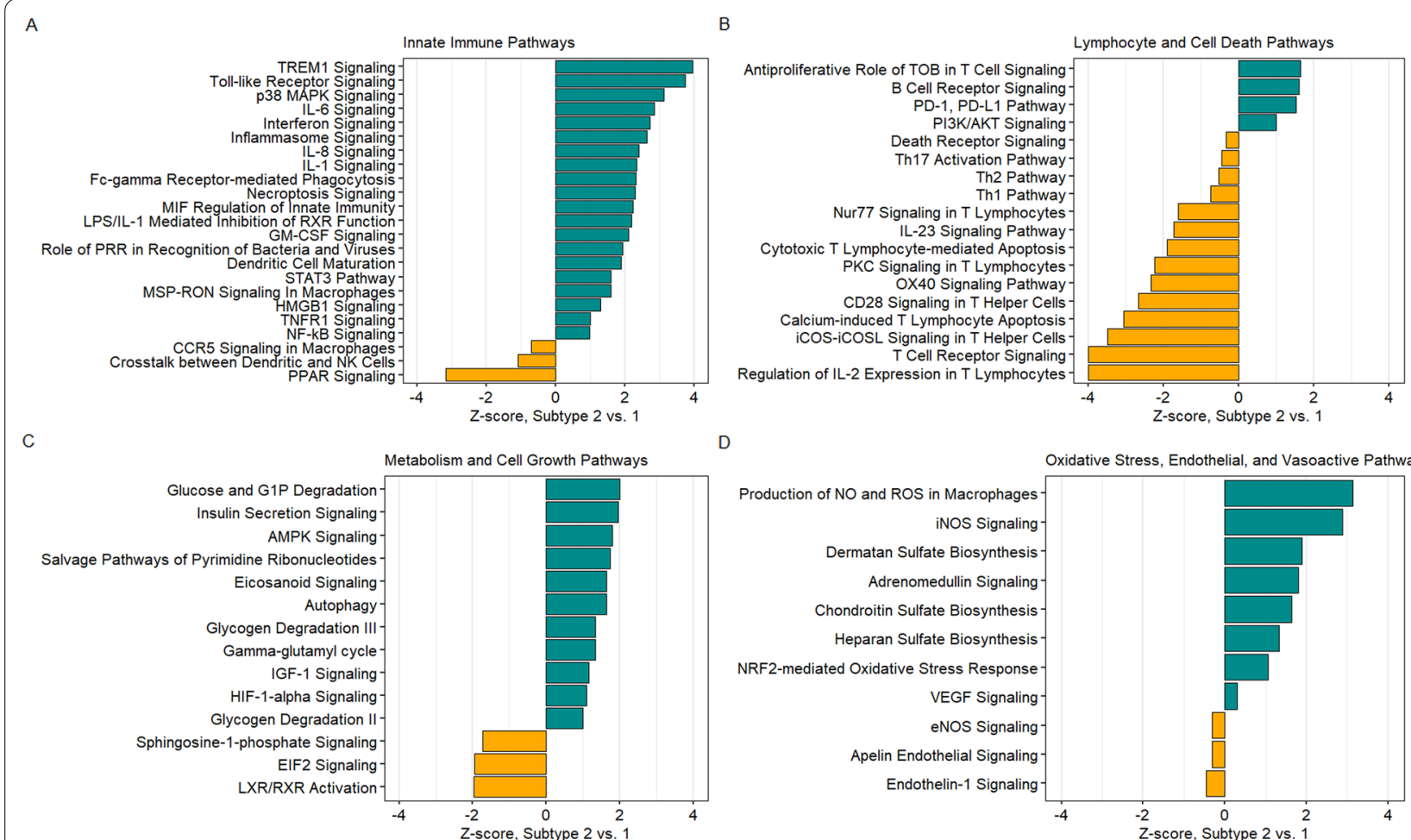

D

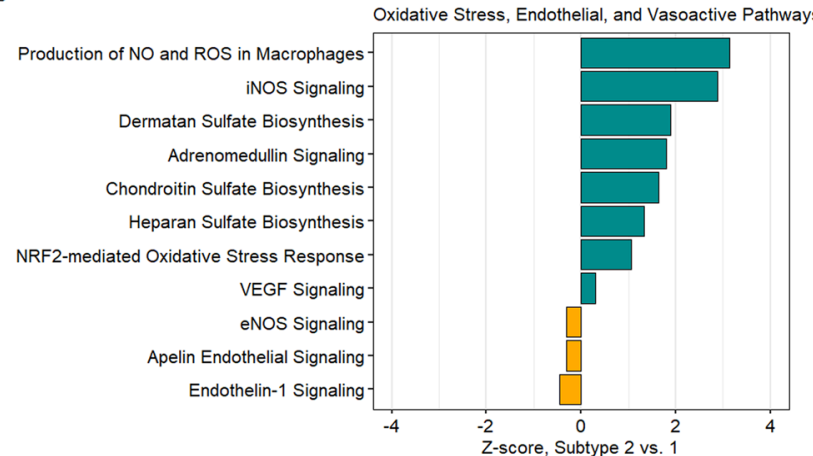

E

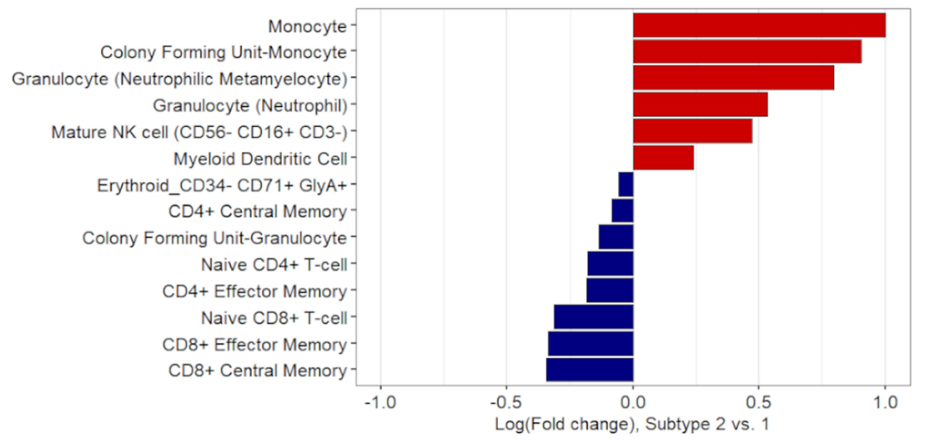

$\mathrm{F}$

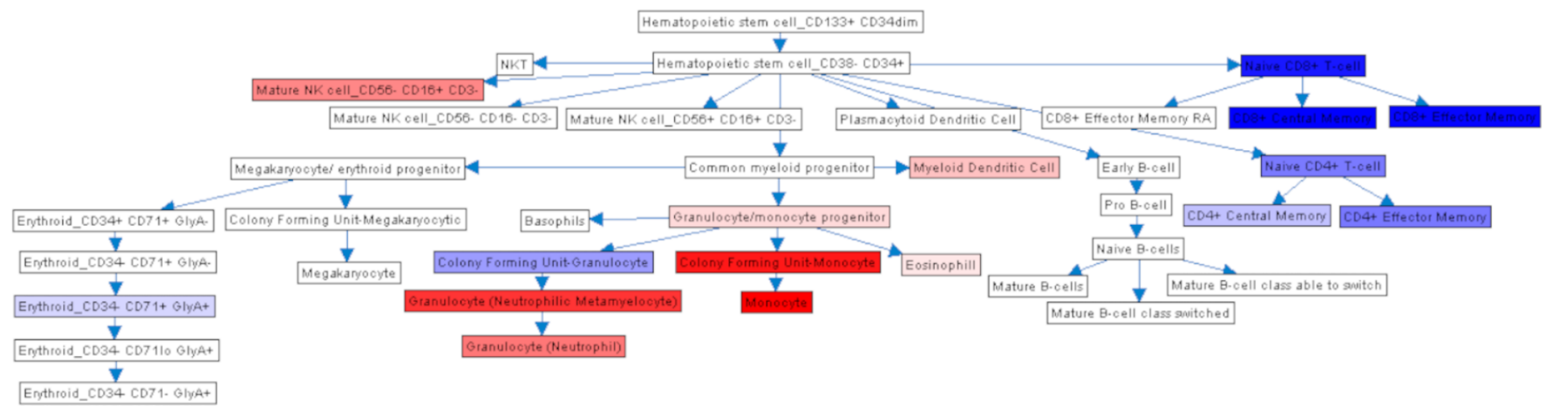

Fig. 4 Biological pathway analysis and immune cell-type deconvolution of transcriptional subtypes. a-d Ingenuity Pathway Analysis of canonical signaling gene sets differentially enriched across transcriptional subtypes based on log-fold change $\geq|1|$ and Benjamini-Hochberg adjusted $p$-value $\leq 0.01 ; Z$-score indicates up-versus down-regulation of signaling gene sets in subtype 2 vs. $1(\mathrm{~N}=128)$. e Relative quantities of immune cell-types inferred across subtypes based on ImmQuant digital cell quantification deconvolution; red shading indicates a higher inferred quantity of cell-type in subtype 2 vs. 1 based on log-fold change; blue shading indicates a lower inferred quantity of cell-type in subtype 2 vs. 1 based on log-fold change $(\mathrm{N}=128)$. $\mathbf{f}$ Hematopoietic lineage plot with relative quantities of immune cell-types inferred across subtypes based on ImmQuant digital cell quantification deconvolution $(N=128)$; intensity of red shading indicates a higher inferred quantity of cell-type in subtype 2 vs. 1 based on log-fold change; intensity of blue shading indicates a lower inferred quantity of cell-type in subtype 2 vs. 1 based on log-fold change 
microvascular-protective endothelial NO (Fig. 4d). In T2, which had higher prevalence of shock, we also observed decreased expression of genes involved in maintaining vascular tone (endothelin-1, apelin, adrenomedullin), with increased expression of genes involved in restoration and maintenance of the endothelial glycocalyx (heparan, dermatan, chondroitin sulfates).

\section{Transcriptional subtypes are differentiated by severe HIV-associated TB, organ dysfunction, and mortality} Consistent with our immune mediator analyses, physiologic derangement was more severe in T2, as indicated by higher MEWS and UVA scores and a greater proportion of patients with qSOFA score $\geq 2$ (Fig. $5 \mathrm{a}-\mathrm{c}$, Additional file 1: Table E7). Compared to T1, patients in T2

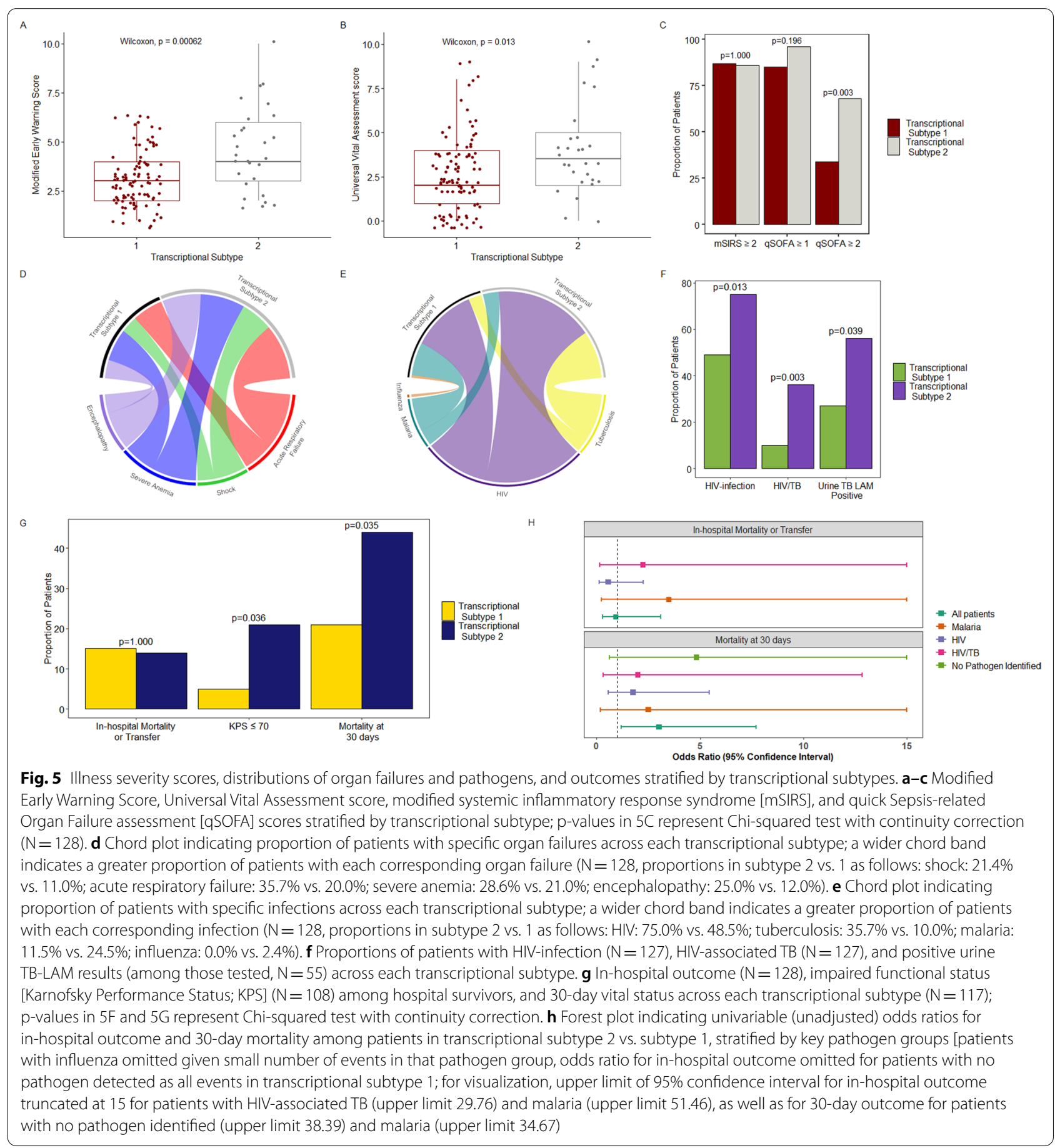


had higher prevalence of shock, acute respiratory failure, severe anemia, and encephalopathy (Fig. 5d, Additional file 1: Table E7). Patients assigned to T2 were predominantly HIV-infected with advanced immunosuppression (HIV clinical stage 3 or 4), severe and often disseminated $\mathrm{TB}$, and less frequent malaria (Fig. 5e, f, Additional file 1: Table E7). Similar to immune mediator subtypes, patients in T2 with positive TB-LAM results had higher band grade intensities than those in T1 (median 3 [IQR 1-3] vs. 1 [IQR 1-1]).

Mortality at 30-days was significantly higher among patients assigned to T2 vs. T1 $(44.0 \%$ vs. $20.7 \%, \mathrm{p}=0.035)$ (Fig. 5g, Additional file 1: Table E8). This finding was consistent when patients with indeterminate 30-day vital status were considered deceased $(50.0 \%$ vs. $27.0 \%$, $\mathrm{p}=0.038$ ), and in multivariable models adjusted for age, sex, illness duration and severity (Additional file 1: Table E8). For patients discharged alive, those in T2 were significantly more likely to have impaired functional status (Fig. $5 \mathrm{~g}$ ). When patients were stratified by pathogen groups, estimates of mortality at 30-days were consistently higher among those in T2 (Fig. 5h).

\section{Subtype-specific prevalence of malaria varies by immune compartment but is consistent for HIV-associated TB}

Among the 122 patients for whom both soluble mediator and RNA-sequencing data were available, 75 (62\%) were assigned to mediator- and transcriptionally-derived subtypes with similar immunological features (i.e. consistently assigned to relatively hyperinflammatory [S2, T2] vs. hypo-inflammatory [S1, T1] immune mediator and transcriptional subtypes) and 47 (38\%) were assigned to dissimilar subtypes (Additional file 1: Figure E8A, Table E9). Although the proportion of HIV-infected patients with more advanced immunosuppression (clinical stages 3 and 4) was highest in T2, prevalence of severe HIV-related infections, including HIV-associated TB, was consistently higher in mediator-derived and transcriptional hyperinflammatory subtypes (Additional file 1: Tables E2-E3, E9 and Figures E8B). In contrast, the prevalence of malaria varied substantially across each approach, with malaria prevalence of $24.5-40 \%$ in mediator-derived hyperinflammatory subtypes (S2) and 11.5\% in the hyperinflammatory, T-cell exhausted transcriptional subtype (T2) (Additional file 1: Table E7 and Figure E8C).

\section{Discussion}

Among adults hospitalized with suspected sepsis in Uganda, we identified distinct immune subtypes defined by differential activation of pro-inflammatory innate and adaptive immune pathways, with transcriptional evidence of T-cell exhaustion, aberrant NK-cell expansion, and hypoxia-induced metabolic reprogramming accompanying the hyperinflammatory subtype. Immune subtypes defined by upregulation of these pathways were consistently associated with severe and often disseminated HIV-associated TB as well as more extensive organ dysfunction and worse clinical outcomes. Our results highlight unique host- and pathogen-driven features of sepsis immunopathology in sub-Saharan Africa, and reinforce the need to develop and test more biologicallyinformed treatment strategies in the region, including those incorporating immunomodulation.

Despite advances towards identifying more precise therapeutic targets and higher-risk, treatment-responsive sepsis subgroups in HICs, biological heterogeneity inherent to sepsis in low-income-settings remains unexplored [5-10, 33, 34]. Here, we show that sepsis host response subtypes differentiated by activation of key immunometabolic pathways are present in a generalizable SSA setting and associated with unique microbiological and prognostic features. If replicated elsewhere, these host subtypes could conceivably be leveraged to inform more locallyrelevant models of sepsis immunopathology and targeted immunomodulatory therapies. For example, while over 50 clinical trials of corticosteroids in sepsis and septic shock have been conducted in HICs (primarily in the context of mitigating potential adrenal insufficiency), none have been conducted in SSA, with guidance on their use in low-income settings driven by expert opinion $[35,36]$. Accordingly, there is a need to further evaluate the role of these low-cost immunomodulatory agents in sepsis management in SSA, in parallel with continued efforts to precisely define therapeutically exploitable pathways.

In HICs, studies comparing sepsis host response profiles among patients with different causative pathogens have reported conflicting results [37]. While several have found host profiles to be consistent across varied microbiological etiologies and sites of infection [3739], others identified source-specific heterogeneity in the host response [40]. Across soluble mediator- and transcriptionally-derived immune subtypes identified in our Ugandan cohort, the prevalence of severe HIV-related infections, including frequently disseminated $\mathrm{TB}$, was consistently higher in those defined by greater pro-inflammatory innate immune activation, aberrant NK-cell expansion, and T-cell exhaustion. Despite the disproportionate burden of sepsis among HIV-infected adults in SSA, little remains known about the immunopathology of HIV-associated sepsis in the region, of which disseminated TB is a leading cause [3, 41, 42]. Independent of critical illness, HIV-infected adults in SSA show evidence of persistent monocyte/ macrophage activation and systemic inflammation, 
some of which persists despite antiretroviral therapy (ART) and viral suppression [43]. HIV-related activation of monocytes/macrophages may also induce of a state of hyperresponsive immune priming that precipitates disproportionate release of pro-versus anti-inflammatory cytokines following pathogen stimulation [32]. Collectively, our observations of a high-risk, HIV-predominant host subtype defined by amplified pro-inflammatory innate immune activation and NKand T-cell exhaustion highlight the need to evaluate the role of immunomodulatory agents in conjunction with prompt initiation or continuation of ART among patients with HIV-associated sepsis in SSA, in parallel with efforts to optimize antimicrobial strategies for TB and other high-burden co-infections [44, 45].

Although the prevalence of severe HIV-associated infections was consistently higher in immune mediator and transcriptionally-derived hyperinflammatory subtypes, subtype-specific prevalence of malaria varied, with higher prevalence in mediator-derived hyperinflammatory subtypes. Nonetheless, patients with malaria assigned to hyperinflammatory subtypes, which showed concomitant endothelial dysfunction, had consistently poorer outcomes. While end-organ sequestration due to microvascular obstruction and endothelial dysfunction are established pathobiological mechanisms in severe malaria, the role of inflammatory mediators is less well-defined [46]. Consistent with our data, prior studies have identified imbalances between pro- and anti-inflammatory mediators among patients with severe falciparum malaria at higher risk of organ dysfunction and death [46, 47]. Although prior trials of immunomodulatory agents in severe falciparum malaria have not shown benefit [48], eventual stratification of trial populations based on immune-inflammatory subtypes could conceivably be used to enhance prognostic and predictive enrichment when evaluating new agents [48].

Our study has limitations. First, our findings are derived from a single-center cohort of medical ward patients and require replication in other geographic and clinical care settings in SSA. Second, although serum mediator samples were available for nearly all patients (96\%), whole-blood RNA samples were available from approximately $43 \%$. Third, as we were unable to isolate peripheral-blood-mononuclear-cells given resource-limitations at our study site, we performed computational deconvolution analyses to infer quantities of immune-cell subsets. Next, although rapid diagnostics for key pathogens were performed in the parent study based on WHO guidelines, we did not perform blood cultures. Lastly, the cross-sectional nature of our sample collection precludes determination of temporal stability of the identified host subtypes.

\section{Conclusions}

We have demonstrated the presence of immunopathologically-distinct and clinically-meaningful host response subtypes among adults with suspected sepsis in Uganda. In conjunction with improvements in acute-care capacity, future studies are needed to refine understanding of sepsis immunopathology in SSA with the goal of developing more locally-relevant, biologically-informed, and clinically-effective management strategies.

\begin{abstract}
Abbreviations
SSA: Sub-Saharan Africa; HIC: High-income countries; EGRH: Entebbe General Referral Hospital; RESERVE-U: Research in the Epidemiology of Severe and Emerging Infections in Uganda; WHO: World Health Organization; IL: Interleukin; IFN: Interferon; IP-10/CXCL10: IFN- $\gamma$-induced-protein-10/C-X-C motifchemokine-10; MIP-1a/CCL3: Macrophage-inflammatory-protein-1-alpha/ chemokine (C-C motif)-ligand 3; MIP-1 $/$ /CCL4: Macrophage-inflammatoryprotein-1-beta/chemokine (C-C motif) ligand-4; TNF-a: Tumor-necrosisfactor-a; Ang: Angiopoietin; MIF: Macrophage-migration-inhibitory-factor; sTNFR1: Soluble-TNF-receptor-type-1; sIL-2Ra/sCD25: Soluble-IL-2-receptoralpha/soluble-CD25; PAI-1: Plasminogen-activator-inhibitor-1; RNA: Ribonucleic acid; qSOFA: Quick-Sepsis-related Organ Failure Assessment; S1: Soluble mediator subtype 1; S2: Soluble mediator subtype 2; UVA: Universal Vital Assessment; MEWS: Modified Early Warning Score; TB: Tuberculosis; TB-LAM: Tuberculosis-Lipoarabinomannan;T1: Transcriptional subtype 1;T2: Transcriptional subtype 2; ART: Anti-retroviral therapy.
\end{abstract}

\section{Supplementary Information}

The online version contains supplementary material available at https://doi. org/10.1186/s13054-022-03907-3.

Additional file 1: Supplemental methods, tables, and figures.

\section{Acknowledgements}

The authors would like to acknowledge and thank the patients enrolled in this study and their families, clinicians at Entebbe General Referral Hospital, and the staff of the UVRI Arbovirology and Emerging and Reemerging Infections Laboratory for their assistance with data and sample collection and laboratory testing.

\section{Authors' contributions}

M.J.C., B.B., and M.R.O'D conceived the study and its design. B.B., J.J.L, J.K., T.B., J.N., N.O., C.N., and M.M. collected, organized and entered clinical data and blood samples and contributed to pathogen diagnostics. S.S., W.W., M.L., M.J.C., and R.T. contributed to laboratory work. M.J.C and A.P. performed statistical analyses. M.J.C., B.B., M.R.O'D., A.P., S.S.S., and W.I.L. contributed to data analysis and interpretation. M.J.C. drafted the manuscript. All authors read and approved the final manuscript.

\section{Funding}

This work was supported by the National Center for Advancing Translational Sciences [UL1TR001873 to Columbia University, sub-award to M.R.O.], the National Institute of Allergy and Infectious Diseases [F32Al147528 to M.J.C], and the MakCHS-Berkeley-Yale Pulmonary Complications of AIDS Research Training (PART) Program (D43TW009607, sub-award to B.B.) from the Fogarty International Center, National Institutes of Health. Additional support was provided by the Stony Wold-Herbert Fund [M.J.C.], Potts Memorial Foundation [M.J.C.], Thrasher Research Fund [M.J.C.], Burroughs Wellcome Fund/American Society of Tropical Medicine and Hygiene [M.J.C.], and DELTAS Africa Initiative [sub-award to M.J.C., B.B.; grant no. 107743]. The DELTAS Africa Initiative is 
an independent funding scheme of the African Academy of Sciences (AAS), Alliance for Accelerating Excellence in Science in Africa [AESA], and supported by the New Partnership for Africa's Development Planning and Coordinating Agency [NEPAD Agency] with funding from the Wellcome Trust [grant no. 107743] and the UK Government. The funders had no role in study design, data analysis and interpretation, manuscript preparation, or decision to publish.

\section{Availability of data and materials}

RNA-sequencing data analyzed in this study are available in the NIH National Center for Biotechnology Information Sequence Read Archive under BioProject accession number PRJNA794277. Other de-identified data will be made available to researchers affiliated with an appropriate institution following mutual signing of a data access agreement and obtainment of necessary ethics approvals. All code is available on request from Drs. Cummings and Price.

\section{Declarations}

\section{Ethics approval and consent to participate}

Each enrolled participant $\geq 18$ years or their surrogate provided written informed consent. This study was approved by ethics committees at Columbia University (AAAR1450), Uganda Virus Research Institute (GC/127/17/0206/582), and Uganda National Council for Science and Technology (HS2308).

\section{Consent for publication}

All authors reviewed and provided consent to publish the work described in this study.

\section{Competing interests}

The authors declare that they have no competing interests.

\section{Author details}

'Division of Pulmonary, Allergy, and Critical Care Medicine, Department of Medicine, Columbia University Vagelos College of Physicians and Surgeons, 622 West 168th St, PH 8E-101, New York, NY 10032, USA. ${ }^{2}$ Center for Infection and Immunity, Mailman School of Public Health, Columbia University, New York, NY, USA. ${ }^{3}$ Department of Arbovirology, Emerging and Re-Emerging Infectious Diseases, Uganda Virus Research Institute, Entebbe, Uganda. ${ }^{4}$ Immunizable Diseases Unit, Uganda Virus Research Institute, Entebbe, Uganda. ${ }^{5}$ Entebbe General Referral Hospital, Ministry of Health, Entebbe, Uganda. ${ }^{6}$ Division of Infectious Diseases, Department of Pediatrics, Vagelos College of Physicians and Surgeons, Columbia University, New York, NY, USA. ${ }^{7}$ Department of Microbiology and Immunology, Albert Einstein College of Medicine, Bronx, NY, USA. ${ }^{8}$ Department of Pathology and Cell Biology, Vagelos College of Physicians and Surgeons, Columbia University, New York, NY, USA. ${ }^{9}$ Department of Epidemiology, Mailman School of Public Health, Columbia University, New York, NY, USA.

\section{Received: 7 December 2021 Accepted: 26 January 2022}

Published online: 08 February 2022

\section{References}

1. Singer M, Deutschman CS, Seymour CW, et al. The third international consensus definitions for sepsis and septic shock (Sepsis-3). JAMA. 2016;315:801-10.

2. Rudd KE, Johnson SC, Agesa KM, et al. Global, regional, and national sepsis incidence and mortality, 1990-2017: analysis for the Global Burden of Disease Study. Lancet. 2020;395:200-11.

3. Lewis JM, Feasey NA, Rylance J. Aetiology and outcomes of sepsis in adults in sub-Saharan Africa: a systematic review and meta-analysis. Crit Care. 2019;23:212.

4. Morton B, Stolbrink M, Kagima W, Rylance J, Mortimer K. The early recognition and management of sepsis in sub-saharan african adults: a systematic review and meta-analysis. Int J Environ Res Public Health. 2018;15:2017.

5. van der Poll T, van de Veerdonk FL, Scicluna BP, Netea MG. The immunopathology of sepsis and potential therapeutic targets. Nat Immunol. 2017;17:407-20.
6. Reddy K, Sinha P, O'Kane CM, Gordon AC, Calfee CS, McAuley DF. Subphenotypes in critical care: translation into clinical practice. Lancet Respir Med. 2020;8:631-43.

7. Davenport EE, Burnham KL, Radhakrishnan J, et al. Genomic landscape of the individual host response and outcomes in sepsis: a prospective cohort study. Lancet Respir Med. 2016;4:259-71.

8. Scicluna BP, van Vught LA, Zwinderman AH, et al. Classification of patients with sepsis according to blood genomic endotype: a prospective cohort study. Lancer Respir Med. 2017;5:816-26.

9. Antcliffe DB, Burnham KL, Al-Beidh F, et al. Transcriptomic signatures in sepsis and a differential response to steroids. Am J Respir Crit Care Med. 2019;199:980-6.

10. Seymour CW, Kennedy JN, Wang S, et al. Derivation, validation, and potential treatment implications of novel clinical phenotypes for sepsis. JAMA. 2019;28(321):2003-17.

11. Cummings MJ, Bakamutumaho B, Owor N, et al. Stratifying sepsis in uganda using rapid pathogen diagnostics and clinical data: a prospective cohort study. Am J Trop Med Hyg. 2021;105:517-24.

12. World Health Organization. IMAI district clinician manual: Hospital care for adolescents and adults. https://www.who.int/hiv/pub/imai/imai2011/ en/. Accessed 6 Jan 2022.

13. Bolger AM, Lohse M, Usadel B. Trimmomatic: a flexible trimmer for Illumina sequence data. Bioinformatics. 2014;30:2114-20.

14. Andrews, S. FastQC: A quality control tool for high throughput sequence data. http://www.bioinformatics.babraham.ac.uk/projects/fastqc/ (2010).

15. Dobin A, Davis CA, Schlesinger F, et al. STAR: ultrafast universal RNA-seq aligner. Bioinformatics. 2013;29:15-21.

16. Liao Y, Smyth GK, Shi W. featureCounts: an efficient general purpose program for assigning sequence reads to genomic features. Bioinformatics. 2014;30:923-30.

17. McLachlan GJ. Cluster analysis and related techniques in medical research. Stat Methods Med Res. 1992;1:27-48.

18. Lê S, Josse J, Husson F. FactoMineR: an R package for multivariate analysis. J Stat Softw. 2008;25:1-18.

19. Charrad M, Ghazzali N, Boiteau V, Niknafs A. NbClust: an R package for determining the relevant number of clusters in a data set. J Stat Softw. 2014;61:1-36.

20. Fruchterman TM, Reingold EM. Graph drawing by force-directed placement. Software Pract Exper. 1991;21:1129-64.

21. Pavlopoulos GA, Secrier M, Moschopoulos CN, et al. Using graph theory to analyze biological networks. BioData Mining. 2011;4:10.

22. Epskamp S, et al. qgraph: network visualizations of relationships in psychometric data. J Stat Softw. 2012;48:1-18.

23. Love MI, Huber W, Anders S. Moderated estimation of fold change and dispersion for RNA-seq data with DESeq2. Genome Biol. 2014;15:550.

24. Price A, Caciula A, Guo C, et al. DEvis: an R package for aggregation and visualization of differential expression data. BMC Bioinformatics. 2019;20:110.

25. Frishberg A, Brodt A, Steuerman Y, Gat-Viks I. ImmQuant: a user-friendly tool for inferring immune cell-type composition from gene-expression data. Bioinformatics. 2016;32:3842-3.

26. Abbas AR, Baldwin D, Ma Y, et al. Immune response in silico (IRIS): immune-specific genes identified from a compendium of microarray expression data. Genes Immun. 2005;6:319-31.

27. Novershtern N, Subramanian A, Lawton LN, et al. Densely interconnected transcriptional circuits control cell states in human hematopoiesis. Cell. 2011;144:296-309.

28. Lawn SD, Gupta-Wright SD. Detection of lipoarabinomannan (LAM) in urine is indicative of disseminated TB with renal involvement in patients living with HIV and advanced immunodeficiency: evidence and implications. Trans R Soc Trop Med Hyg. 2016;110:180-5.

29. Shah M, Martinson NA, Chaisson RE, Martin DJ, Variava E, Dorman SE. Quantitative analysis of a urine-based assay for detection of lipoarabinomannan in patients with tuberculosis. J Clin Microbiol. 2010;48:2972-4.

30. Lawn SD, Kerkhoff AD, Vogt M, Wood R. Clinical significance of lipoarabinomannan detection in urine using a low-cost point-of-care diagnostic assay for HIV-associated tuberculosis. AIDS. 2012;26:1635-43.

31. Björkström NK, Ljunggren HG, Sandberg JK. CD56 negative NK cells: origin, function, and role in chronic viral disease. Trends Immunol. 2010;31:401-6. 
32. Huson MA, Grobusch MP, van der Poll T. The effect of HIV infection on the host response to bacterial sepsis. Lancet Infect Dis. 2015;15:95-108.

33. Stanski NJ, Wong HR. Prognostic and predictive enrichment in sepsis. Nat Rev Neph. 2020;16:20-31.

34. DeMerle KM, Angus DC, Baillie JK, et al. Sepsis Subclasses: A Framework for Development and Interpretation. Crit Care Med. 2021;49:748-59.

35. Annane D, Bellissant E, Bollaert PE, et al. Corticosteroids for treating sepsis in children and adults. Cochrane Database Syst Rev. 2019; 12:CD002243.

36. Mer M, Schultz MJ, Adhikari NKJ, et al. Core elements of general supportive care for patients with sepsis and septic shock in resource-limited settings. In Sepsis Management in Resource-limited Settings (ed. Dondorp A, Dünser M, Schultz M), Springer, 2019. https://library.oapen.org/handle/ 20.500.12657/22905.

37. Goh C, Knight JC. Enhanced understanding of the host-pathogen interaction in sepsis: new opportunities for omic approaches. Lancet Respir Med. 2017;5:212-23.

38. Pereverzeva L, Uhel F, Sengers HP, et al. Blood leukocyte transcriptomes in gram-positive and gram-negative community-acquired pneumonia. Eur Respir J. 2021;26:2101856. https://doi.org/10.1183/13993003.01856-2021.

39. Burnham KL, Davenport EE, Radhakrishnan J, Humburg P, Gordon AC, Hutton P, et al. Shared and distinct aspects of the sepsis transcriptomic response to fecal peritonitis and pneumonia. Am J Respir Crit Care Med. 2017;196:328-39.

40. Peters-Sengers $\mathrm{H}$, Butler JM, Uhel F, et al. Source-specific host response and outcomes in critically ill patients with sepsis: a prospective cohort study. Intensive Care Med. 2022;48:92-102.

41. Cummings MJ, O'Donnell MR. Inverting the pyramid: increasing awareness of mycobacterial sepsis in sub-Saharan Africa. Int J Tuberc Lung Dis. 2015;19:1128-34.

42. Barr DA, Lewis JM, Feasey N, et al. Mycobacterium tuberculosis bloodstream infection prevalence, diagnosis, and mortality risk in seriously ill adults with HIV: a systematic review and meta-analysis of individual patient data. Lancet Infect Dis. 2020;20:742-52.

43. Kroeze S, Wit FW, Rossouw TM, et al. Plasma biomarkers of human immunodeficiency virus-related systemic inflammation and immune activation in sub-saharan africa before and during suppressive antiretroviral therapy. J Infect Dis. 2019;220:1029-33.

44. Schutz C, Barr D, Andrade BB, et al. Clinical, microbiologic, and immunologic determinants of mortality in hospitalized patients with HIV-associated tuberculosis: A prospective cohort study. PLoS Med. 2019; 16:e1002840

45. Bruyn ED, Fukutani KF, Rockwood N, et al. Inflammatory subtype of patients with tuberculosis with or without HIV-1 co-infection: a prospective cohort study and immunological network analysis. Lancet Microbe. 2021;2:e375-85.

46. Higgins SJ, Kain KC, Liles WC. Immunopathogenesis of falciparum malaria: implications for adjunctive therapy in the management of severe and cerebral malaria. Expert Rev Anti Infect Ther. 2011;9(80):3-19.

47. Day NP, Hien TT, Schollaardt T, et al. The prognostic and pathophysiologic role of pro- and anti-inflammatory cytokines in severe malaria. J Infect Dis. 1999;180:1288-97.

48. Varo R, Erice C, Johnson S, et al. Clinical trials to assess adjuvant therapeutics for severe malaria. Malar J. 2020;19:268.

\section{Publisher's Note}

Springer Nature remains neutral with regard to jurisdictional claims in published maps and institutional affiliations.

Ready to submit your research? Choose BMC and benefit from:

- fast, convenient online submission

- thorough peer review by experienced researchers in your field

- rapid publication on acceptance

- support for research data, including large and complex data types

- gold Open Access which fosters wider collaboration and increased citations

- maximum visibility for your research: over $100 \mathrm{M}$ website views per year

At BMC, research is always in progress.

Learn more biomedcentral.com/submissions 